\title{
Improving visual analyses and communications of ontology by dynamic tree (case study: computer system)
}

\author{
Ameen Shaheen*, Rizik Al-Sayyed, Azzam Sleit \\ Faculty of Computer Science, University of Jordan, Amman, Jordan
}

\section{A RT ICLE INFO}

\section{Article history:}

Received 24 January 2017

Received in revised form

8 April 2017

Accepted 15 April 2017

\section{Keywords:}

Data visualization

Ontology

Tree map

\begin{abstract}
A B S T R A C T
Visual Analysis is one participatory tool for mapping out main domains like ontology; along with their causes and effects, it supports domain planners to identify clear and manageable communications between the components of the domain and the strategy aimed at achieving them. Presenting computer system is a good area of employing visualization effectively; a computer system domain consists of hardware components that are integrated with each other to build full computer. The purpose of this paper is to create a visual analysis dynamic tree for the hardware components of a computer system by creating a number of concepts that represent the knowledge of this domain in a dynamic way in order to reduce the size of the layout since issue is critical in data visualization. The paper also aims at supporting the sharing and the reusing of the represented knowledge on other related problems.
\end{abstract}

(C) 2017 The Authors. Published by IASE. This is an open access article under the CC BY-NC-ND license (http://creativecommons.org/licenses/by-nc-nd/4.0/).

\section{Introduction}

Human has a long history with essential information representation, and data visualization is still an interesting issue today. The historical backdrop of visualization was formed to some degree by accessible innovation and by the squeezing needs of the time, they include: primitive depictions on muds, maps on dividers, photos, table of numbers (rows and columns) (Card et al., 1999).

Visualization is a central component of revelation, comprehension, and communication in science. It is additionally the way toward changing concepts, numbers, and objects into a shape that is obvious to the human eyes (Healy and Moody, 2014).

There are wide ranges of approaches to see. Visualization tools extend from false-shading photos of adjustable pictures in cosmology to recreations of ancient animals in fossil science. A lot of visualization tools and techniques have turned out to be accessible to support activities such as modeling, testing and analysis. There is ample recounted prove that suitable visualization can altogether reduce the exertion spent on these activities (Harel, 1992).

One of the most important tools is a tree-map which Imagine the task of dealing with the execution

\footnotetext{
* Corresponding Author.

Email Address: Ameen1223@gmail.com (A. Shaheen) https://doi.org/10.21833/ijaas.2017.05.011

2313-626X/C) 2017 The Authors. Published by IASE.

This is an open access article under the CC BY-NC-ND license

(http://creativecommons.org/licenses/by-nc-nd/4.0/)
}

of a hundred compressors to minimize generation downtime.

Tree-map helps in this procedure by giving a theoretical visual representation of the compressors of enthusiasm for a single screen show that can be gathered and organized by geographic district, resource group, field or platform (Plaisant et al., 2003).

On other hand, ontology is an investigation of conceptions of reality and the nature of being. It is the science of what is, of the kinds and structures of the properties, objects and relations in every area of reality (Genesereth and Nilsson, 1987).

So, ontology are defines the kinds of things that exist in an application domain in a hierarchy structure way. But they do that in a statically fashion as we will see later (Sleit et al., 2011).

In this paper we will use the domain of computer hardware components since it has many parts that connected to each other's to make a full computer. we will build the ontology for this domain and also we will build it by the tree map and shows the cons and pros for each of them to describe what our improvement has do.

The rest of this paper is organized as follows: in Section 2, gives a general view of the ontology and why we need it, Tree-map is illustrated in Section 3. While in Section 4, computer hardware components will be presented and how can visualize by using ontology and tree-map. Moreover, In Section 5, our work will be shown and how we improve the visual analysis and the communications between the 
domain components. Finally, the conclusions will be discussed in Section 6.

\section{Ontology}

The word "Ontology" is utilized with various implications as a part of various groups. The term ontology has been utilized for various years by artificial intelligence and knowledge representation group. However, is currently turning out to be a piece of the standard terminology of a much more extensive group of information systems modeling. The term is obtained from philosophy, where ontology implies a systematic account of existence (Guarino et al., 2009).

Ontology is "the specification of conceptualizations" used to help projects and people to share knowledge. Aristotle managed this subject in his Metaphysics and defined Ontology as the exploration of "being qua being," for example, the investigation of attributes that have a place with things as a result of their extremely nature as shown in Fig. 1. Not at all like the trial sciences, which go for finding and demonstrating reality under a specific point of view, has Ontology concentrated on the nature and structure of things, freely of any further considerations, and even autonomously of their real presence (Roussey et al., 2011).

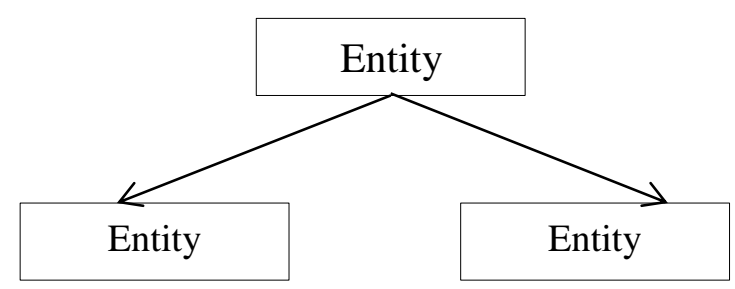

Fig. 1: Ontology structure

Now, the question is why might somebody need to build up ontology?. In study of Natalya and Deborah (2001) they give the answer. A portion of the reasons are:

- To share regular understanding of the structure of data among individuals or software agents.

- To make area suppositions express.

- To break down domain knowledge.

- To empower reuse of domain knowledge.

- To separate domain information from the operational knowledge.

Since ontology have a certain number of advantages such like improve reusability and interoperability, improvement on searches, improvement of navigation and they can permit inferences but also it have a number of disadvantages (Sleit et al., 2008). the main issue is the Visualization problems because of it is the size of the resource is inversely proportional to its specificity so, to if we want to describe a big domain this is mean we need a large space which is not allowed in data visualizations. And it does not give enough information for each part of the domain. This is one of the most issues that we will try to solve it in our work.

\section{Tree-map}

Tree-map is one of visualization tools that first appear in 1990 in response to the common problem of a filled hard disk, it became with the idea of producing a compact visualization of directory tree structures as shown in Fig. 2 (Shneiderman and Plaisant, 1998).

Tree-Maps are utilized to display hierarchical data on 2D or 3D shows. Tree-maps offer many features such like clients can visualize as well as manipulate categorized and various algorithms are known for tree-maps specifically, Mixed tree-maps, Binary tree, slice and dice etc. (Kong et al., 2010).

Tree-map are used when the space is important and when we have a large amount of hierarchical data that we need to get an overview of it.

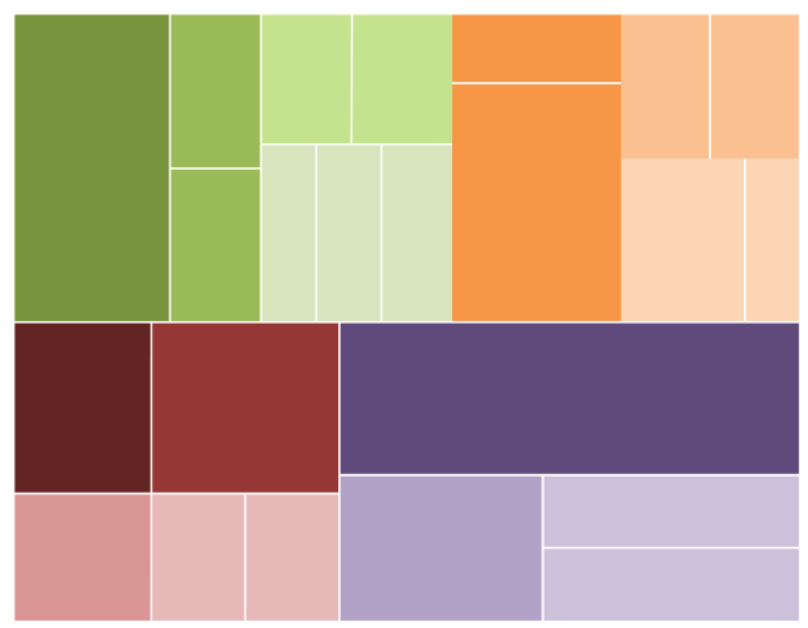

Fig. 2: Tree-map

Tree maps should primarily be used with values that can be aggregated.

As an advantage of the Tree-Map is economical in that they can be utilized inside a constrained space but then show an extensive number of things at the same time.

At the point when there is a connection amongst shading and size in the tree structure, we can see designs that would be hard to spot in different ways, for instance, when a specific color is especially important (Jadeja and Shah, 2015).

On the other hand the disadvantages, Tree-maps are bad when there is a major contrast in the greatness of the measure values and also it doesn't give enough information about the data that displayed inside it.

Since the size is also the most important issue for the map tree do display a large data (Salah et al., 2005), from this point we focus on how we can combine the concept of the ontology and the Treemap to create a dynamic tree to improve the visual analysis and communications in the domain by visualize the data on hierarchical tree but the tree 
will be created in dynamic way in the same place to reduce the area size and to arrangement the parts in clear display way to be easy to understand.

\section{Computer hardware components domain}

Computer Hardware is the physical part of a computer, as distinguished from the computer software that executes or runs on the hardware. Computer Hardware Components has many parts that connected to each other's to make a full computer.

By using the top-down method we divided the concepts of the CHC (Computer Hardware Components) into three main parts:

1) $\mathrm{CPU}$

2) Memory

3) I/O devices

The classes above have a subclasses and a set of instances for example: Memory class is divided to two subclasses which are: Main and Secondary Memory. By using the concept of ontology we can use it to show the domain of computer hardware components as shown in Fig. 3.

Fig. 3 is a hierarchical graph of the ontology Computer Hardware Components concepts. Which the rectangles are represent the concepts and edges are represent the relations.

Since the ontology describes the computer hardware components domain but we can see the drawback of the size and also there is no information about each component this will make the reader can't understand or this can lead to misunderstand for the general audience (Shaheen and Sleit, 2016).

On the other hand, we can use the tree-map to visualize the domain of commuter hardware components as shown in Fig. 4. But as we mentioned before the Tree-Maps are utilized to display hierarchical data on 2D or 3D shows and its offer many features such like clients can visualize as well as manipulate categorized but also the same issue of the ontology is appear here because of we can't provide an enough information for each part of the domain and the data are displayed in a static way which can't give the user the ability to understand the relations (communications) between the components.

\section{Dynamic tree}

In this part we will describe how the dynamic tree will improve the visual analysis and communications of the ontology.

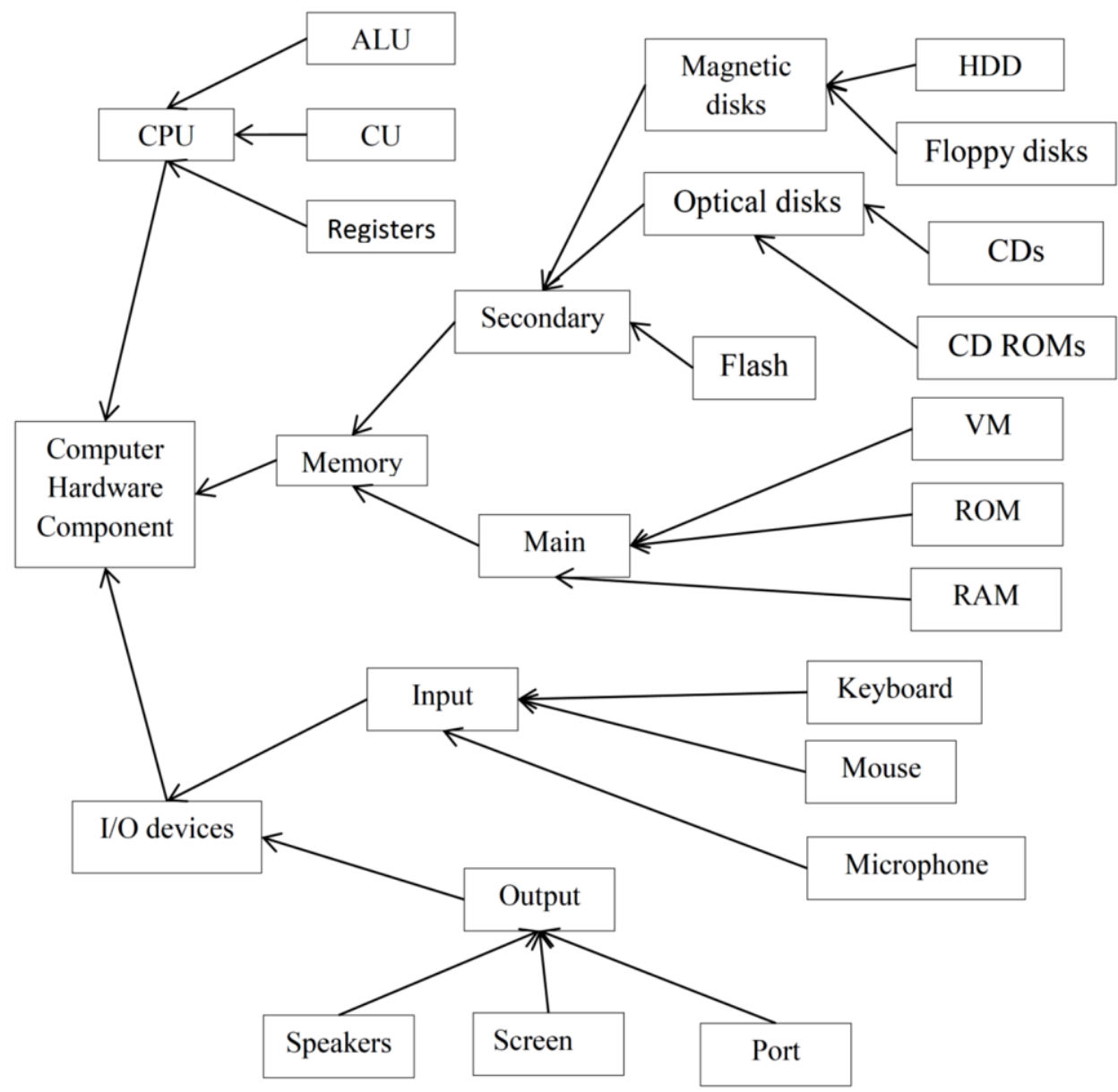

Fig. 3: Ontology of computer hardware components

We create a dynamic-tree by visualize the data on hierarchical tree but the tree will be created in a dynamic way in the same place to reduce the area size because of it is very critical in data visualization 
as we mention before and to arrangement the parts in clear display way to be easy to understand. Also, for each part of the domain we provide a brief description about to make it understandable for any one that read the domain.

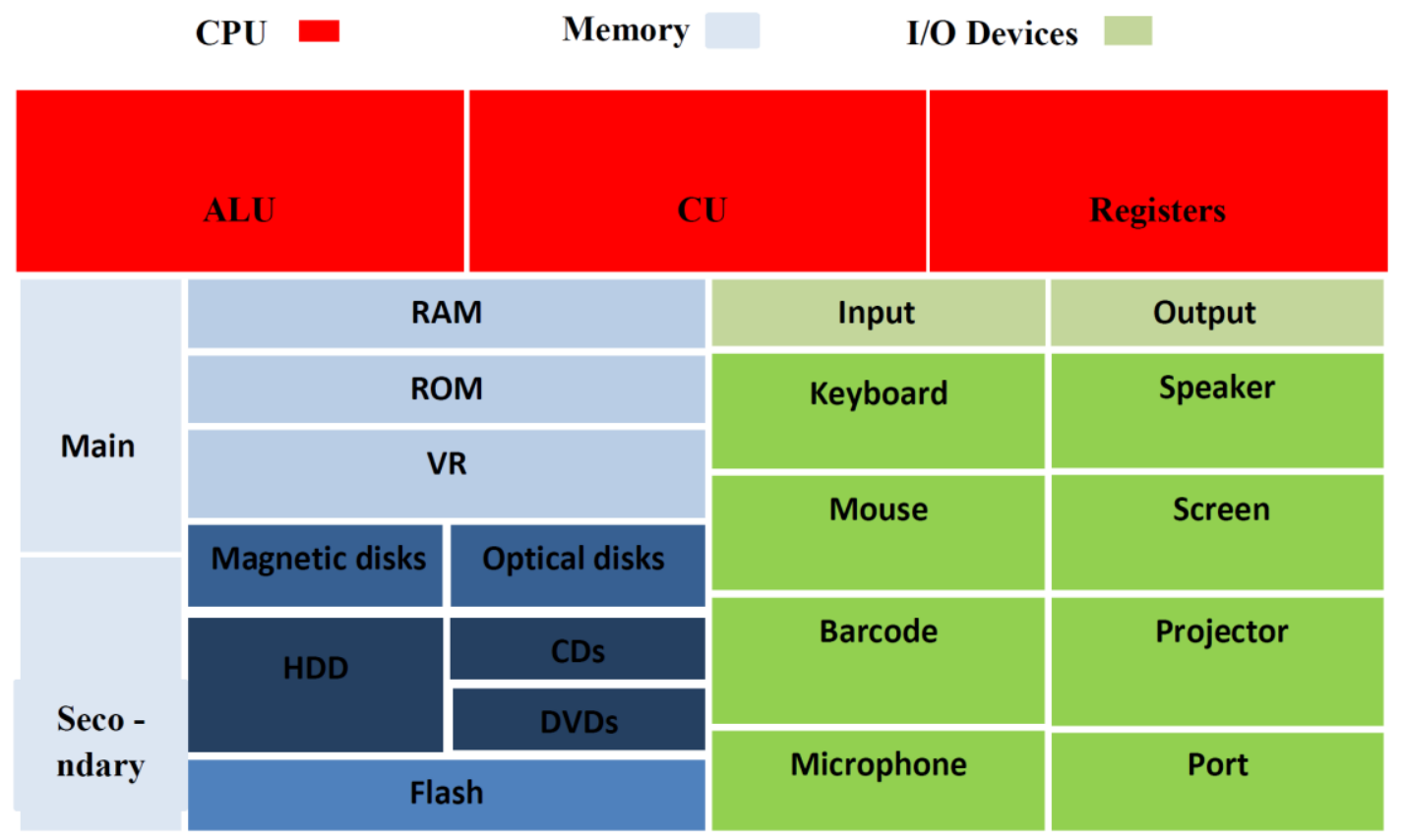

Fig. 4: Tree-map of computer hardware component

We got the idea from the advantages of both (tree-map and ontology) by describe the domain in hierarchical way like the ontology style and we use the color to shows the relations between the components as the tree map style.

In Fig. 5, we can see how the components of the computer hardware displayed and how we provide information about each part.
Part A in Fig. 5 Introduce the key components of computer hardware and on click on each component the tree will go directly to next level of selected component as mention in part B, Fig. 5 shows a three level of the memory component.

We developed the dynamic tree by using HTML5 and java script language, we give each main class a unique color, then when we go in depth, the color will be more dark as shown in Fig. 5.

Computer Hardware components

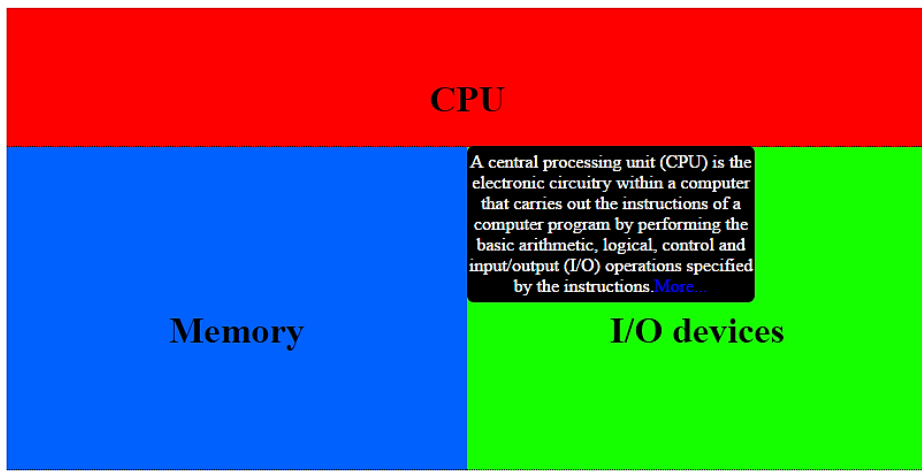

(a)

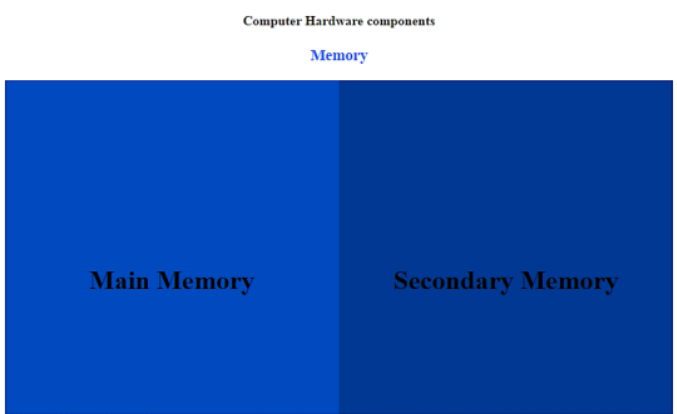

(b)

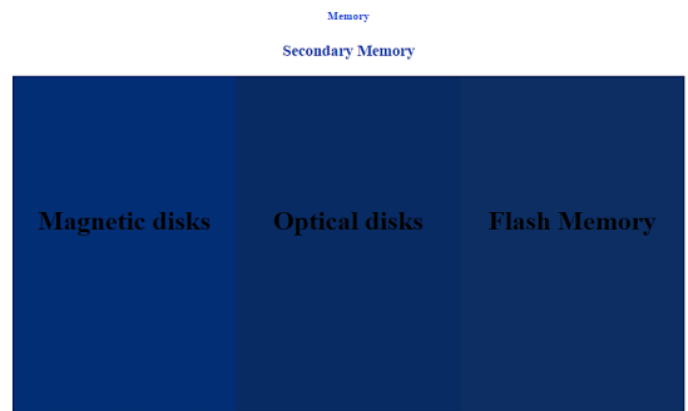

(c)

Fig. 5: Dynamic tree for computer hardware component (a) the root level, (b) level 1 in memory, and (c) level 2 in memory 
Also, for each part, when the user put the mouse on any concept the dynamic tree will provide a little information and also at the end there is a more word which on click will redirect to the full information about this part.

\section{Conclusion}

We defined the concepts of ontology and treemaps with how to represent each of them in specific domain like the domain of computer hardware components and their advantages and disadvantages. We create the dynamic tree to improve the visual analysis and communications in the ontology and map-tree by introduce the components of domain dynamically (in-place) to reduce the display space since it is the most critical thing in data visualization and to provide additional information to readers which will improve the sharing and reuse of the represented knowledge among others related problems.

\section{References}

Card SK, Mackinlay JD, and Shneiderman B (1999). Readings in information visualization: using vision to think. Morgan Kaufmann, Burlington, USA.

Genesereth MR and Nilsson NJ (1987). Logical foundations of artificial Intelligence. Morgan Kaufmann, San Mateo, USA.

Guarino N, Oberle D, and Staab S (2009). What is an ontology?. In: Staab S and Studer R (Eds.), Handbook on Ontologies: 1-17. Springer, Heidelberg, Germany

Harel D (1992). Biting the silver bullet: Toward a brighter future for system development. Computer, 25(1): 8-20.

Healy K and Moody J (2014). Data visualization in sociology. Annual Review of Sociology, 40: 105-128.
Jadeja M and Shah K (2015). Tree-map: A visualization tool for large data. SIGIR Workshop on Graph Search and Beyond, Santiago, Chile: 9-13.

Kong N, Heer J and Agrawala M (2010). Perceptual guidelines for creating rectangular tree-maps. IEEE Transactions on Visualization and Computer Graphics, 16(6): 990-998. https://doi.org/10.1109/TVCG.2010.186

Natalya FN and Deborah LMG (2001). Ontology development 101: A guide to creating your first ontology. Technical Report SMI2001-0880, Stanford Medical Informatics. Available online at: http://liris.cnrs.fr/alain.mille/enseignements/Ecole_Centrale /What\%20is\%20an\%20ontology\%20and\%20why\%20we\%2 0need\%20it.htm

Plaisant C, Chintalapani G, Lukehart C, Schiro D, and Ryan J (2003). Using visualization tools to gain insight into your data. In the SPE Annual Technical Conference and Exhibition. Society of Petroleum Engineers Inc., Denver, USA: 1-9.

Roussey C, Pinet F, Kang M, and Corcho $O$ (2011). An introduction to ontologies and ontology engineering. In: Falquet G, Métral C, Teller J, and Tweed C (Eds.), Ontologies in Urban Development Projects: 9-38. Springer Science \& Business Media, Berlin, Germany.

Salah I, Sleit A, Al-Sharaeh S, Huneiti A, and Obeed N (2005). Efficient method for assigning students to proper groups. Editorial Advisory Board e, 21(2): 249-358

Shaheen A and Sleit A (2016). Comparing between different approaches to solve the $0 / 1$ Knapsack problem. International Journal of Computer Science and Network Security, 16(7):110.

Shneiderman B and Plaisant C (1998). Tree maps for spaceconstrained visualization of hierarchies. Available online at: http://www.cs.umd.edu/hcil/treemap-history.

Sleit A, Qatawneh M, Al-Sharief M, Al-Jabaly R, and Karajeh 0 (2011). Image clustering using color, texture and shape features. KSII Transactions on Internet \& Information Systems, 5(1): 211-227.

Sleit A, Salah I, and Jabay R (2008). Approximating images using minimum bounding rectangles. In the $1^{\text {st }}$ International Conference on the Applications of Gigital Information and Web Technologies (ICADIWT'08), IEEE: 394-396. https://doi.org/10.1109/ICADIWT.2008.4664379 